\title{
DUVFEL PHOTOINJECTOR DYNAMICS: MEASUREMENT AND SIMULATION
}

\author{
W.S. Graves, L.F. DiMauro, R. Heese, E.D. Johnson, J. Rose, J. Rudati, T. Shaftan, B. Sheehy, \\ L.-H. Yu, NSLS, Brookhaven National Laboratory, Upton, NY 11973 \\ D. H. Dowell, Boeing Physical Sciences Research Center, Seattle, WA 98124
}

\begin{abstract}
The DUVFEL photoinjector consists of a 1.6 cell BNL gun IV with copper cathode, variable pulse length Ti:Sapp laser, and solenoid magnet. The beam dynamics and the electromagnetic fields in the photoinjector have been characterized by producing a short electron beam with very low charge that is used as a field probe. Transverse beam size and divergence are measured as a function of initial RF phase and initial spot size and compared with simulations using the code HOMDYN. The electromagnetic fields used in the simulations are produced by SUPERFISH, and have been verified with RF measurements. The simulations and measurements of beam dynamics are presented.
\end{abstract}

\section{MEASUREMENT METHOD}

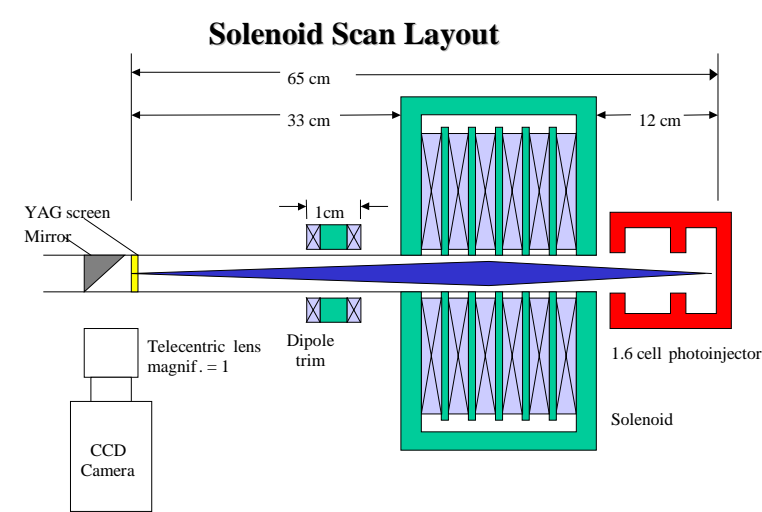

Figure 1: Layout of solenoid scan emittance measurement experiment. Note short distances.

Measurements of beam size, divergence, and emittance from a S-band photoinjector [1] are performed using the solenoid scan method. The experimental layout is indicated in Fig. 1. The solenoid scan is very similar to the quadrupole scan emittance measurement [2], except that coupling of $\mathrm{x}$ and $\mathrm{y}$ must be accounted for. In matrix transport notation the beam envelope evolves according to

$$
\sigma_{1}=R \sigma_{0} R^{T}
$$

where $\sigma$ and $\mathrm{R}$ are $4 \times 4$ matrices. It is desireable to decouple $\mathrm{x}$ and $\mathrm{y}$ so that in the horizontal plane

$$
\left\langle x_{1}^{2}\right\rangle=R_{11}^{2}\left\langle x_{0}^{2}\right\rangle+2 R_{11} R_{12}\left\langle x_{0} x_{0}^{\prime}\right\rangle+R_{12}^{2}\left\langle x_{0}^{2}\right\rangle
$$

To achieve this, multiply both the R-matrix and sigmamatrix by the rotation matrix $\mathrm{T}$.

$$
\begin{aligned}
& T=\left(\begin{array}{cccc}
\cos \theta & 0 & \sin \theta & 0 \\
0 & \cos \theta & 0 & \sin \theta \\
-\sin \theta & 0 & \cos \theta & 0 \\
0 & -\sin \theta & 0 & \cos \theta
\end{array}\right) \\
& \theta=\frac{e B L}{2 m m c^{2}}=\arctan \frac{R_{31}}{R_{11}} \\
& R \rightarrow T R \\
& \sigma \rightarrow T \sigma
\end{aligned}
$$

Note that this rotation is applied to the measured second moments of the beam, rather than to the full beam image. This is valid for simple, symmetric beam distributions such as gaussian or uniform shapes. Irregular shapes with structure would require rotation of the full image.

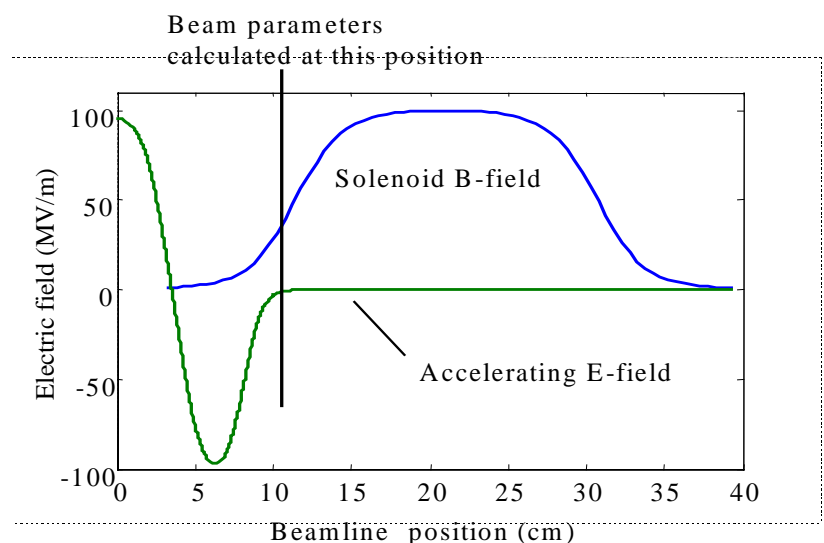

Figure 2:Plots of RF field and focusing solenoid field. Twiss parameters are calculated at the gun exit, in the fringe field of the solenoid.

The R-matrix for the solenoid is found by slicing it into 40 hard-edge solenoids [3] that accurately follow the measured field profile. Because the solenoid field extends into the gun, a plane is chosen slightly inside the solenoid, and just outside the acccelerating fields, to reconstruct the beam's second moments in both measurement and simulation.

The calculated R-matrix accurately accounts for the beam transport in the absence of space charge effects and correlations due to RF forces. These effects are found to be negligible [4] for charge less than $7 \mathrm{pC}$ and bunch length less than 5 ps FWHM. Under these conditions the 
electron pulse is a useful probe of the electric fields in the RF cavity. For different initial RF phases and different initial spot sizes the beam divergence and size provide a measure of the integrated transverse fields in the cavity. These measurements are then compared with simulation.

\section{LASER AND ELECTRON PULSE PARAMETERS}
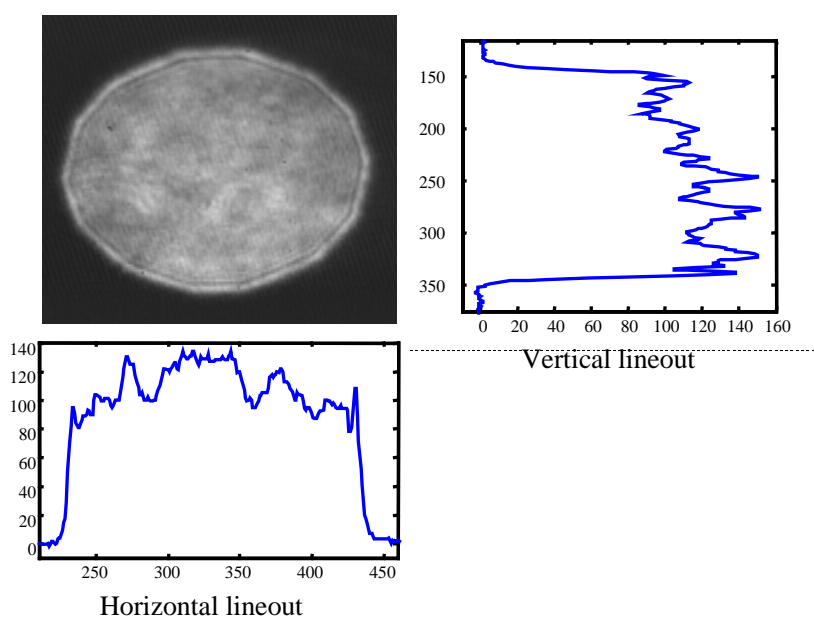

Figure 3: Laser beam spatial profile showing horizontal and vertical lineouts through beam centroid.

Less than $5 \mathrm{uJ}$ of $\mathrm{UV}$ are required to produce a few $\mathrm{pC}$ of charge for the photoinjector studies. Spatial variations in the UV light are filtered with a pinhole aperture, and the resulting distribution's wings are clipped with an iris. The iris location is fourier imaged to the cathode. Figure 3 shows an image at a complimentary location to the cathode. This image indicates a hard-edge distribution with good uniformity.

The time profile of the UV pulse is measured (Fig. 4) with a Hamamatsu FESCA 500 streak camera. This camera has sub-ps resolution for soft IR photons, but hard UV photons produce energetic electrons in the camera's micro-channel plate which degrade its resolution to about 2.4 ps. The time profile of the electron beam is verified using the RF-zero phasing method [5,6]. The measured electron pulse length is $2.6 \mathrm{ps}$. At an initial RF phase of 30 degrees, simulations indicate that the electron pulse is compressed a few percent relative to the laser pulse, so that this is also a good estimate of the laser pulse length.

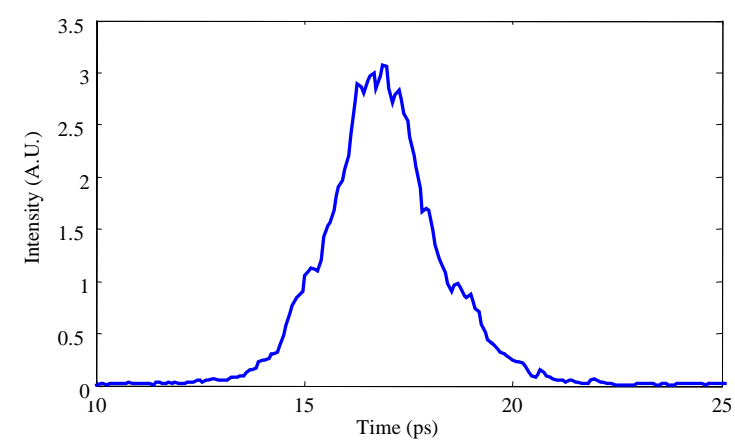

Figure 4: Time profile of UV laser pulse measured with streak camera. $\mathrm{FWHM}=2.8 \mathrm{ps}$.

\section{MEASUREMENT VS SIMULATION}

Software has been developed that provides for rapid experimental analysis during beam studies. Figure 5 shows the user interface to the framegrabber and analysis software. The MATLAB software environment is used to run a PC-based 8-bit video framegrabber and to perform analysis and plotting. Video processing includes subtraction of background (dark current) images, application of a $3 \mathrm{X} 3$ median filter to remove hot pixels due to bremstrahlung $\mathrm{X}$-rays, and zeroing of all pixels that are less than a user settable percentage of the maximum pixel value. A single emittance measurement takes about

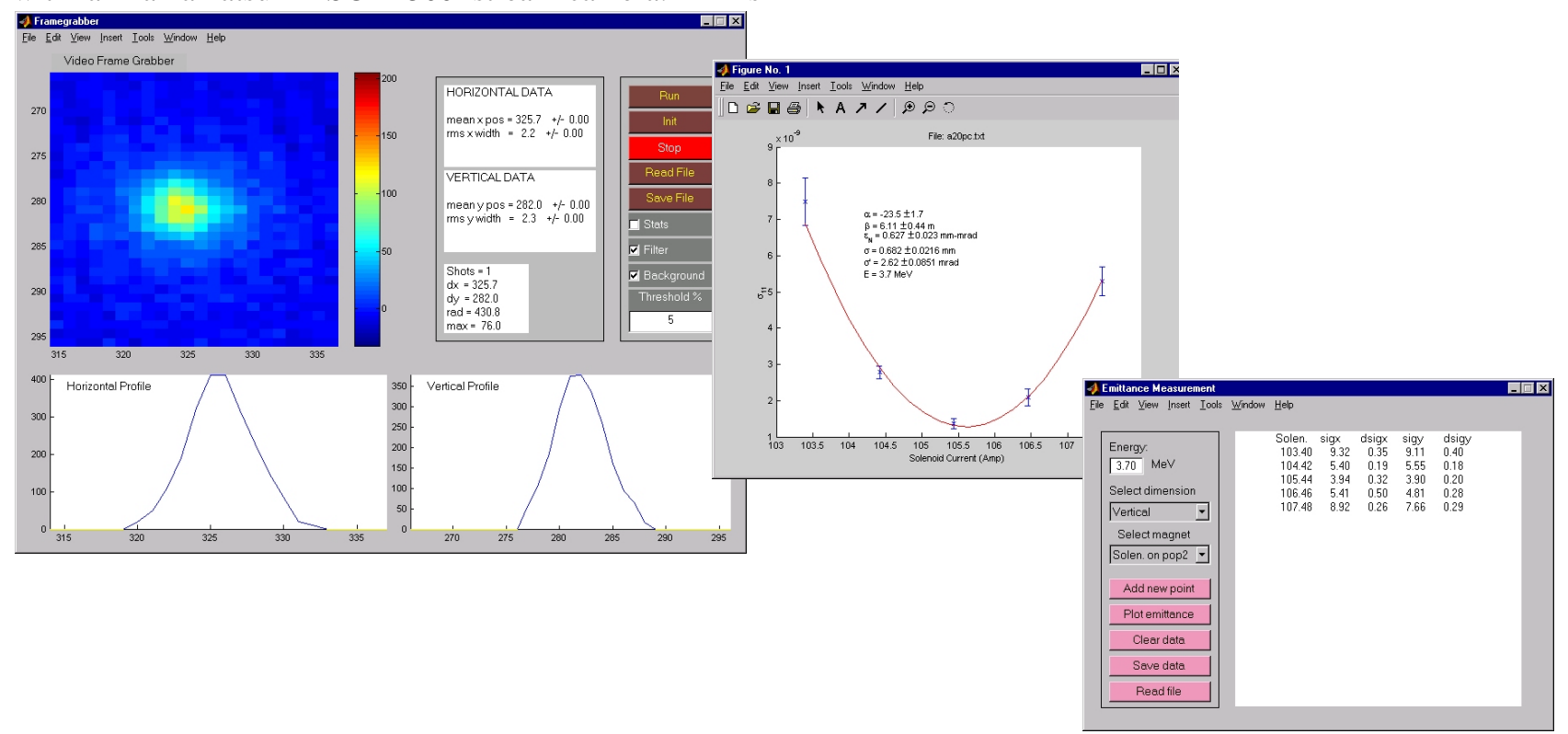

Figure 5: User interface allows near real-time analysis of data and enables rapid exploration of parameter space. 
2 minutes. As reported elsewhere [4] the emittance measurements are in agreement with theoretical estimates of the thermal emittance. This measured emittance is entered in the simulations as an input condition.

The RF fields of the cavity are simulated with SUPERFISH. Two models are used: one with the field balance set so that the $1 / 2$ cell field is $10 \%$ higher than the full cell, and one with it set $10 \%$ lower. The fields generated by these models are input into the HOMDYN beam dynamics code [7] along with the measured solenoid magnetic field. An overall scaling of the field is done by measuring the beam energy at initial RF phase of 30 degrees, then scaling the SUPERFISH field to produce the same energy at that phase. This is due to the difficulty of precise measurements of the delivered RF power.

HOMDYN transports the simulated beam from the cathode surface to the gun exit including the effects of space charge. The pulse is longitudinally divided into thin time-slices, and each slice evolves according to the local forces.
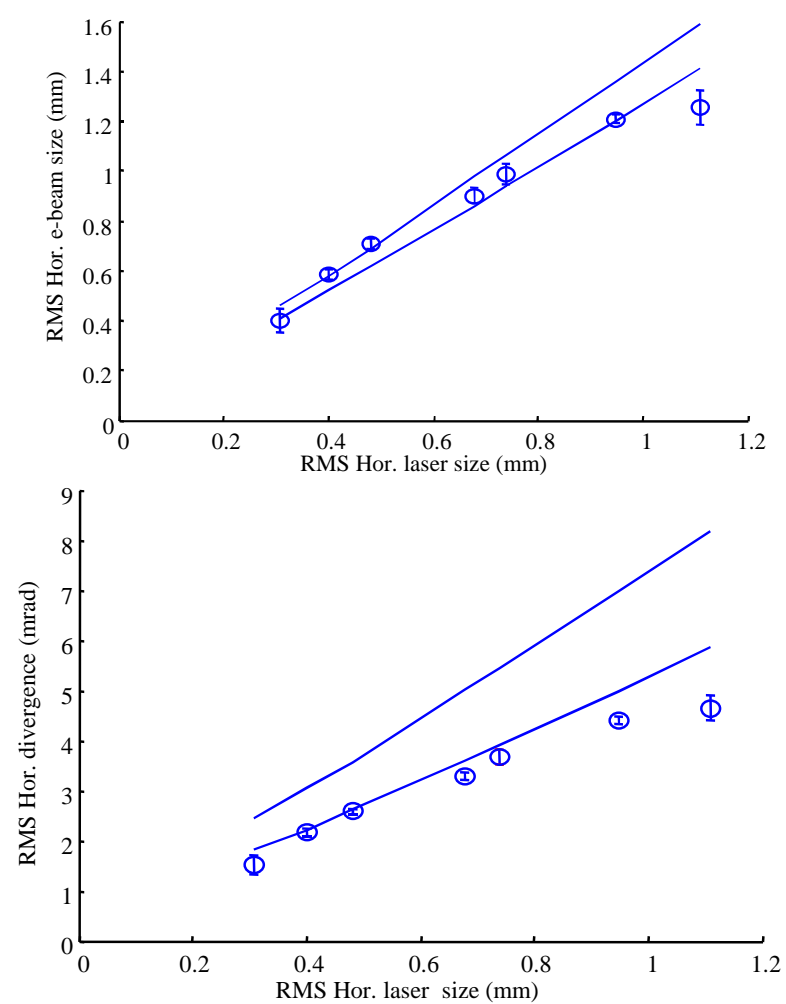

Figure 6: Horizontal beam size and divergence vs laser spot size. See text for details.

Fig. 6 shows plots of the measured horizontal beam size and divergence (data points with error bars) vs initial laser size. The two bounding curves are the simulation results from HOMDYN. The high $1 / 2$ cell field imbalance is indicated by the upper line, and the low $1 / 2$ cell field imbalance is indicated by the lower line. After substantial retuning of the field balance and rework of the cathode [8], the good agreement between simulation and measurement was obtained. It was found that the initial cathode design was subject to arcs and perhaps field emission that limited the $1 / 2$ cell field to a maximum value, and thereby lowered the beam divergence at low charge at the gun exit. At high charge, the opposite effect occurs, where the strong space-charge field causes a larger than expected divergence due to the weak acceleration in the $1 / 2$ cell. Fig. 7 shows the beam divergence before and after the cathode upgrade.

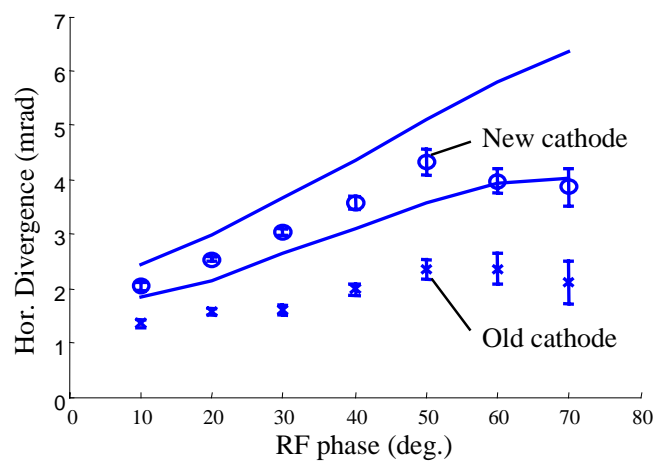

Figure 7: Beam divergence vs initial RF phase before and after cathode redesign.

\section{CONCLUSIONS}

A short, low charge beam has been used to probe the $\mathrm{RF}$ fields of a photoinjector using the solenoid scan emittance measurement method. Good agreement between measured beam parameters and HOMDYN simulations is found after modifications were performed to the experiment.

We thank J. Barry, P. Marino, and P. Singh for expert technical assistance.

\section{REFERENCES}

[1] D.T. Palmer et al, in Proceedings of the 1997 Particle Accelerator Conference (Vancouver, B.C., 1997), p. 2687. [2] M.C. Ross et al, in Proceedings of the 1987 IEEE Particle Accelerator Conference (Washington, D.C., 1987) p. 725.

[3] V. Yakimenko, private communication.

[4] W.S. Graves et al, "Measurement of Thermal Emittance for a Copper Photocathode", in these proceedings (Chicago, IL, 2001).

[5] D.X. Wang and G.A. Krafft, in Proceedings of the 1997 Particle Accelerator Conference (Vancouver, B.C., 1997), p. 2020.

[6] W.S. Graves et al, "Ultrashort Electron Bunch Length Measurements at the DUVFEL", in these proceedings (Chicago, IL, 2001).

[7] M. Ferrario et al, SLAC-PUB-8400, LNF-00-004 (2000)

[8] J. Rose et al, "Modeling and Measurements of the DUVFEL Photoinjector Cavity RF Properties", in these proceedings (Chicago, IL, 2001). 